\section{Neural networks in the future of neuroscience research}

\author{
Mikail Rubinov
}

Neural networks are increasingly seen to supersede neurons as fundamental units of complex brain function. In his Timeline article (From the neuron doctrine to neural networks. Nat. Rev. Neurosci. 16, 487-497 $(2015))^{1}$, Yuste provides a timely overview of this process, but does not clearly differentiate between biological neural network models (broadly and imprecisely defined as empirically valid models of (embodied) neuronal or brain systems, which enable the emergence of complex brain function through distributed computation) and artificial neural network models (a relatively well-defined class of networks originally designed to model complex brain function ${ }^{2}$ but now mainly viewed as a class of biologically inspired data-analysis algorithms useful in diverse scientific fields ${ }^{3}$ ).

A distinction between biological and artificial neural network models is important as the neuroscience network paradigm is mainly driven by the aim of uncovering biologically valid mechanisms of neural computation. Artificial neural networks were initially proposed as candidate models for such computation but, despite being enthusiastically researched at the end of the twentieth century, they have largely not bridged the gap between elegant theory and neuroscientific observation ${ }^{4,5}$. In this context,
Yuste's emphasis on some classic artificial neural network models does not seem to be supported by the evidence of, or the promise for, the problem-solving capacity of these models in neuroscience ${ }^{6}$.

What could be an alternative promising approach to biologically valid neural network modelling? At present we can only speculate, but the ongoing development of highresolution high-throughput brain imaging technologies - including those being developed as part of the BRAIN Initiative ${ }^{7}-$ and the consequent availability of increasingly large structural ${ }^{8}$ and functional ${ }^{9}$ imaging data sets, make it appealing to initially search for patterns in such data in less theorybound and more data-driven ways ${ }^{10,11}$, and to subsequently construct theories a priori constrained on these discovered patterns ${ }^{12}$. A famous example of this approach in biology is the formulation of the theory of evolution by natural selection; this theory arose from an initial aim to catalogue all living biological organisms on earth, and from a subsequent careful analysis of the obtained diverse biological data ${ }^{13}$. Interestingly, artificial neural networks may yet prove to be important in this quest but in the role of powerful tools for analysing complex imaging data sets ${ }^{14}$, rather than as a theoretical foundation for how the brain computes.
Mikail Rubinov is at the Department of Psychiatry and Churchill College, University of Cambridge, Cambridge CB3 ODS, UK; and the Janelia Research Campus, Howard Hughes Medical Institute, Ashburn, Virginia 20147, USA

e-mail:mr572@cam.ac.uk

doi: $10.1038 / \mathrm{nrn} 4042$ Published online 21 October 2015

1. Yuste, R. From the neuron doctrine to neural networks. Nat. Rev. Neurosci. 16, 487-497 (2015).

2. Rumelhart, D. E., McClelland, J. L. \& The PDP Research Group. Parallel Distributed Processing: Explorations in the Microstructure of Cognition (MIT Press, 1986).

3. LeCun, Y., Bengio, Y. \& Hinton, G. Deep learning. Nature 521, 436-444 (2015).

4. Marcus, G. in The Future of the Brain: Essays by the World's Leading Neuroscientists (eds Marcus, G. $\&$ Freeman, J.) 205-215 (Princeton Univ. Press, 2014).

5. Zador, A. in The Future of the Brain: Essays by the World's Leading Neuroscientists (eds Marcus, G. \& Freeman, J.) 40-49 (Princeton Univ. Press, 2014).

6. Laudan, L. Progress and Its Problems: Towards a Theory of Scientific Growth (University of California Press, 1978).

7. Alivisatos, A. P. et al. Nanotools for neuroscience and brain activity mapping. ACS Nano 7, 1850-1866 (2013).

8. Oh, S. W. et al. A mesoscale connectome of the mouse brain. Nature 508, 207-214 (2014).

9. Ahrens, M. B. et al. Brain-wide neuronal dynamics during motor adaptation in zebrafish. Nature 485, 471-477 (2012).

10. Sporns, O. Discovering the Human Connectome (MIT Press, 2012).

11. Vogelstein, J. T. et al. Discovery of brainwide neuralbehavioral maps via multiscale unsupervised structure learning. Science 344, 386-392 (2014).

12. Sejnowski, T. J., Churchland, P. S. \& Movshon, J. A. Putting big data to good use in neuroscience. Nat. Neurosci. 17, 1440-1441 (2014).

13. Kell, D. B. \& Oliver, S. G. Here is the evidence, now what is the hypothesis? The complementary roles of inductive and hypothesis-driven science in the postgenomic era. BioEssays 26, 99-105 (2004).

14. Helmstaedter, M. et al. Connectomic reconstruction of the inner plexiform layer in the mouse retina. Nature 500, 168-174 (2013).

\section{Acknowledgements}

The author thanks C. Chang for helpful comments. The author has received funding from the NARSAD Young Investigator Award, the Isaac Newton Trust Research Grant and the Parke Davis Exchange Fellowship.

Competing interests statement

The author declares no competing interests 